\section{IN THE NEWS}

Memories are made of ads

Have you ever had your memory of a cherished - or not-so-cherished childhood event called into question by a parent who points out that it actually happened to your brother? If so, you will sympathize with the subjects of experiments that hit the headlines after being described by Elizabeth Loftus of the University of Washington at the British Association Science Festival in Glasgow, UK.

"The advertisers are coming for your childhood"

The Independent

(UK, 5 September 2001)

"No thanks for the memory ... it was only a TV advert" The Guardian

(UK, 5 September 2001)

There is plenty of evidence that our memories are less reliable than we think they are. For example, if you have been told repeatedly that a certain event happened in your childhood, you are likely to end up 'remembering' it - even though the memory has been created by your brain in response to the retelling of the story. Such 'false memories' can seem just as vivid as the real thing. Loftus carried out experiments to show that adverts can also rewrite our childhood memories. When they were shown a fake advert for Disneyland that featured children shaking hands with Bugs Bunny, people in her study were more likely to say that they remembered meeting Bugs Bunny at Disneyland during their childhood - even though Bugs Bunny isn't a Disney character.

So the next time an advert prompts you to go out looking for that sweet you loved as a child, or that coffee your mother always used to buy, beware - you could be the unwitting victim of memory manipulation by the advertisers.

\title{
A question of timing
}

Just as a person's character is believed to be shaped by their birth order relative to their siblings, so the identity of many neurons is determined by the sequence in which they are generated. The mechanisms that control this temporal mode of patterning are just beginning to be understood, and as reported in Cell, Isshiki et al. have gained important new insights into a molecular mechanism that enables neurons in Drosophila to 'remember' their birth order.

The Drosophila neural precursor cells, or neuroblasts, undergo a series of asymmetric cell divisions, budding off smaller ganglion mother cells (GMCs), most of which divide once more to generate two neurons. Neurons that are born early populate the deep cell layers and develop long axonal processes, whereas later-born neurons colonize increasingly more superficial layers and develop shorter processes. Birth order is also reflected in the expression of transcription factor genes; early-born neurons express the hunchback gene $(h b)$, neurons in the middle layers express $p d m$ and late-born neurons express castor (cas). In addition, Isshiki et al. have identified a population of cells between the $h b$ - and $p d m$ expressing layers that express the Krüppel (Kr) gene. The authors analysed the expression of these four genes in neuroblasts during development, and showed that, irrespective of neural lineage, they are almost invariably expressed transiently in neuroblasts, following the sequence $h b \rightarrow K r \rightarrow p d m$ $\rightarrow$ cas. The GMCs and their neuronal progeny inherit and stabilize the gene expression profile of the neuroblast at the time of their birth. In loss- and gain-of-function experiments, Isshiki et al. showed that the expression of birth order genes in GMCs is both necessary and sufficient to specify birth order identity (on the basis of gene expression, neurotransmitter expression and/or axon morphology). However, although the fate of cells expressing specific genes is invariant in a given lineage, there is considerable variation between lineages. For example, $h b$-expressing GMCs can give rise to motor neurons, interneurons or even glia, depending on the neuroblast from which they arise.

This study raises some intriguing questions. For example, how does the expression of the genes become stabilized in the GMCs to create a birth-order 'memory'? It also remains to be seen how the expression sequence is regulated. Isshiki et al. found evidence of regulation within the pathway, with each factor able to activate the next gene in the sequence while repressing the next-but-one gene. However, this is unlikely to be the main controlling mechanism, as inactivation of any one gene causes only minor perturbations in the expression sequence. The authors suggest the involvement of an additional clock mechanism that is linked to the cell cycle.

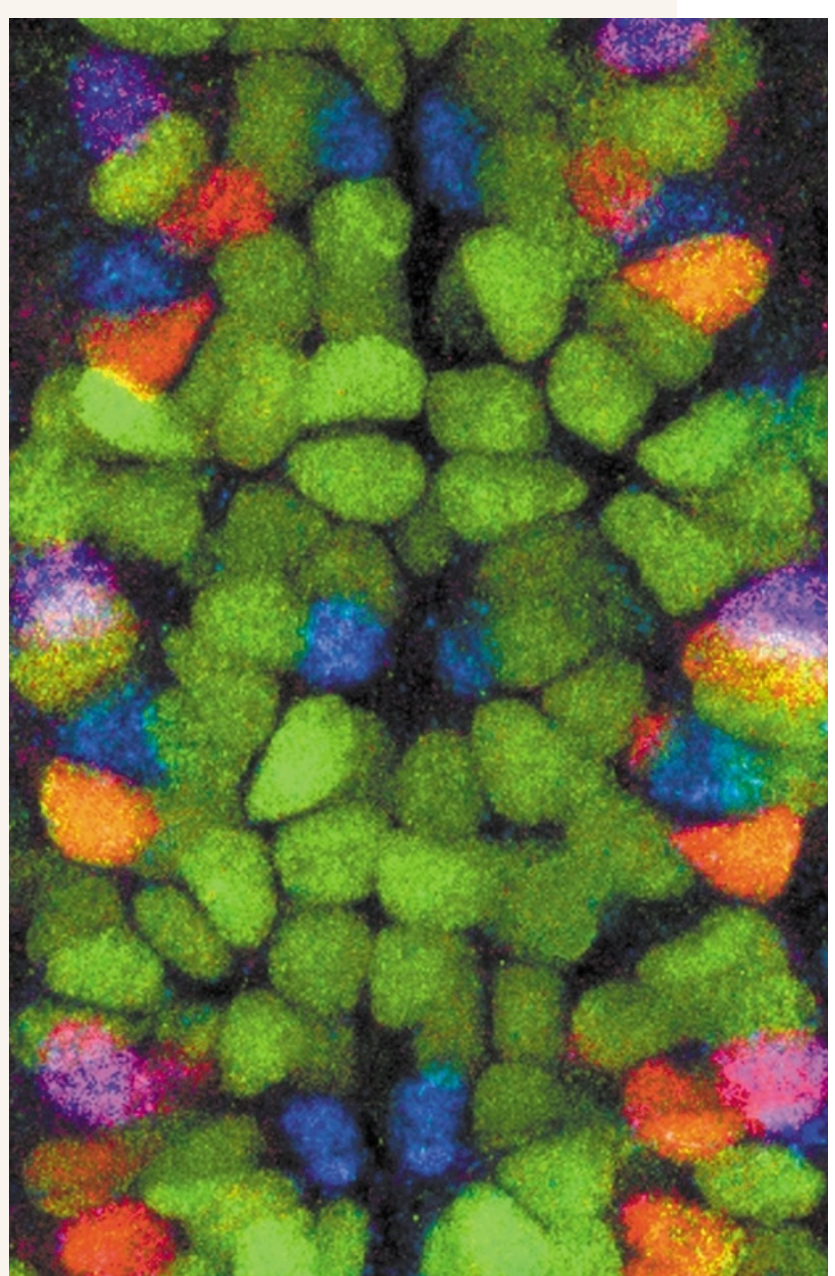

Triple-labelled confocal image of a field of Drosophila neuroblasts that are cell-cycle arrested by a mutation in the string (cdc25) gene. Neuroblasts are stained for Krüppel (green), Pdm1 (blue) and Eagle (red). Photograph by Bret Pearson, University of Oregon, USA.

In certain regions of the vertebrate nervous system, including the cortex and the retina, cells are generated in a highly stereotyped temporal sequence. Mammalian homologues of some of the Drosophila birth order genes have already been identified, and future studies should show whether temporal patterning in vertebrates is controlled by a similar mechanism to that described by Isshiki and colleagues.

Heather Wood

69) References and links

ORIGINAL RESEARCH PAPER Isshiki, T. et al. Drosophila neuroblasts

sequentially express transcription factors which specify the tempora identity of their neuronal progeny. Cell 106, 511-521 (2001)

FURTHER READING Livesey, F. J. \& Cepko, C. L. Vertebrate neural cellfate determination: lessons from the retina. Nature Rev. Neurosci. $\mathbf{2}$, 109-118 (2001)

WEB SITE

Chris Doe's lab: http://www.neuro.uoregon.

edu/doelab/doelab.html 